\title{
Prosodic emphasis versus word order in Greek instructive texts
}

Christina Alexandris and Stavroula-Evita Fotinea

Institute for Language and Speech Processing (ILSP), Athens, Greece

https://doi.org/10.36505/ExLing-2006/01/0010/000010

\begin{abstract}
In Greek instructive texts, the production of clear and precise instructions is achieved by both prosodic modelling (Alexandris, Fotinea \& Efthimiou, 2005) and morphosyntactic strategies (Alexandris, 2005). In the set of experiments performed in the present study, we attempt to determine whether prosodic modelling or whether morphosyntactic strategies, namely word order, constitutes the most crucial factor in the production of Greek spoken instructions most clearly and correctly understood by the receiver. In the present set of experiments, emphasis will be given in respect to utterances containing temporal expressions. The set of experiments performed in the present study involves the contrastive comparison and evaluation of 36 written and spoken utterances containing the temporal expressions, placed in different syn-tactic positions.
\end{abstract}

\section{Word order in technical manuals and prosodic emphasis in task-oriented dialog systems}

In the present study we attempt to determine whether the perceived prominence of elements in a sentence is primarily defined by prosody or by word order. The results of this experiment will contribute to the determination of whether prosodic modelling (1) or whether morphosyntactic strategies, namely word order (2), constitutes the most crucial factor in the production of Greek instructions most clearly and correctly understood by the receiver. The set of experiments performed will focus on utterances containing temporal expressions. Temporal and spatial expressions, as observed in recorded corpora of spoken Greek instructive texts, constitute the third largest group of elements receiving prosodic emphasis (after quantifiers and numerical expressions) and prosodic emphasis on temporal expressions is observed to produce a more precise and restrictive reading than when the same element in the same phrase is not emphasized (Alexandris, Fotinea \& Efthimiou, 2005). In written Greek instructive texts such as technical manuals, usually involving a relatively high degree of Information Management (Hatim, 1997), ambiguities in respect to the semantics of spatial and temporal representation are solved with morphosyntactic strategies such as Rephrasing (Alexandris, 2005). Rephrasing is a controlled-language like approach (Lehrndorfer, 1996), where the key-word of the sentence, usually a negation,

ExLing 2006: Proceedings of 1st Tutorial and Research Workshop on Experimental Linguistics, 28-30 August 2006, Athens, Greece 
a sublanguage-specific expression or a spatial and temporal expression, is positioned in the beginning of the sentence or phrase (Alexandris, 2005).

\section{Word order versus prosodic emphasis in temporal expressions \\ Experiment}

The set of experiments performed in the present study involves the evaluation of 18 written and 18 corresponding spoken utterances containing the temporal expressions, placed in different syntactic positions. The precise meaning of the spoken utterances containing elements of prosodic emphasis will be compared with the same set of corresponding written utterances. The written utterances were evaluated before the corresponding spoken utterances. The spoken utterances were evaluated in a separate session. The evaluation was performed by 30 native speakers (Standard Greek), both male $(43,33 \%)$ and female $(56,67 \%)$ (ages $25-45)$. In the first set, the respondents were to indicate the most important element of each sentence. The second set of sentences involved spoken sentences in which the respondents were requested to repeat exactly the same process. The sets of utterances were retrieved from an instructive text, namely a technical manual accompanying a coffee machine as a professional appliance. The corpus of written and spoken utterances consisted of permutations of simple and compound sentences containing temporal expressions placed in various grammatically acceptable and equally distributed positions before or after the verb, deverbal noun or entire sentence modified. Temporal expressions or phrases containing temporal expressions were placed in grammatically correct positions, and also in accordance to semantic acceptability, so as not to bias the respondents in their evaluation.

\section{Results}

In the set of spoken sentences, the results indicated that $93,7 \%$ of the respondents selected the prosodically emphasized temporal expressions as the most important element in the sentence. In contrast to the spoken utterances, the results obtained from the set of written sentences presented very diverse and rather equally distributed results (Table 1). Specifically, in the set of written utterances, only $10 \%$ of the respondents marked all 18 phrases containing the temporal expressions that fully coincided with the prosodically emphasized temporal expressions in the set of spoken sentences. However, it should be noted that all respondents selected at least one written utterance with a temporal expression. 
Table 1. Results for the Spoken and Written Utterances.

\begin{tabular}{|l|l|l|}
\hline \multicolumn{2}{|l|}{ Percentages of respondents } & Written Utterances \\
\hline $\begin{array}{l}\text { Number of utterances with } \\
\text { Temporal Expression selected }\end{array}$ & Spoken Utterances & \\
\hline 18 out of 18 & $93,7 \%$ & $0,0 \%$ \\
\hline $17-18$ out of 18 & $0,0 \%$ & $10,0 \%$ \\
\hline $14-16$ out of 18 & $0,0 \%$ & $26,6 \%$ \\
\hline $11-13$ out of 18 & $0,0 \%$ & $20,0 \%$ \\
\hline $5-10$ out of 18 & $0,0 \%$ & $13,3 \%$ \\
\hline $1-4$ out of 18 & $6,3 \%$ & $20,0 \%$ \\
\hline
\end{tabular}

The second most commonly marked element in the set of written utterances is observed to be the subjunctive na-clause (Table 2), a complement clause with infinitival behaviour (Giannakidou, 2005). The respondents' selection of the na-clauses, occurring in 16 out of the 18 utterances, was equally distributed among the different syntactic structures of the utterances. Thus, we observed no evident relation between na-clause selection and the position of the na-clause in respect to the temporal expression or the phrase containing the temporal expression. A third group comprised elements containing nouns with qualitative modifiers such as "golden filter" and "medium grinded powder". These sentences often occurred within the marked naclauses, but were also often individually marked by some respondents. Further investigation is necessary to determine whether the criteria for the marking of the na-clauses are primarily syntactically or semantically based.

Table 2. An utterance with a na-clause (underlined).

\begin{tabular}{|c|c|}
\hline 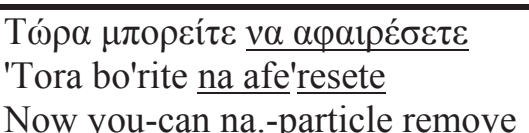 & 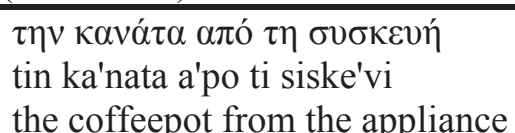 \\
\hline
\end{tabular}

Table 3. Results for the most commonly marked element.

\begin{tabular}{|l|l|l|}
\hline \multicolumn{3}{|l|}{ Percentages of Written Utterances selected by Respondents } \\
\hline Number of utterances & temporal expressions & na-clauses \\
\hline All (18) & $10,0 \%$ & $0,0 \%$ \\
\hline 10 and over $(10-17)$ & $46,6, \%$ & $13,3 \%$ \\
\hline 5 and over $5-9)$ & $33,3 \%$ & $20,0 \%$ \\
\hline Less than 5 (4- 1$)$ & $26,6 \%$ & $30,0 \%$ \\
\hline None $(0)$ & $0,0 \%$ & $20,0 \%$ \\
\hline
\end{tabular}

The obtained data indicates that in written utterances, the class of elements considered to require special attention by the reader is perceived dif- 
ferently among the respondents. The present data shows that temporal expressions do to tend to be of semantic prominence to the receiver (Table 3), however, the data also shows that this phenomenon involving temporal expressions is not consistent and varies from respondent to respondent and other elements of the utterance may be considered semantically more important.

\section{Conclusion}

The present data indicates that prominence of elements in written utterances may be perceived differently among the respondents and further studies are necessary to investigate whether perceived prominence is syntactically or semantically determined. On the other hand, prosodic prominence is equally perceivable to most respondents since, in the spoken utterances, the temporal expressions, constituting the prosodically emphasized elements, were considered by almost all respondents to constitute the semantically most important element of the sentence. Therefore, according to the data obtained from the present study in Greek, prosodic modelling (1) constitutes the most crucial factor in the production of instructions most clearly and correctly understood by the receiver. In contrast, morphosyntactic strategies, namely word order (2) play a secondary role. The relation between syntax and semantics in respect to the determination of the most important element in written utterances constitutes an area of further research.

\section{References}

Alexandris, C. 2005. English as an intervening language in manuals of Asian industrial products: Linguistic Strategies in technical translation for less-used European languages. In Proceedings of the Japanese Society for Language Sciences JSLS 2005, Tokyo, Japan, 91-94.

Alexandris, C., Fotinea, S-E., Efthimiou, E. 2005. Emphasis as an Extra-Linguistic Marker for Resolving Spatial and Temporal Ambiguities in Machine Translation for a Speech-to-Speech System involving Greek. In Proceedings of HCII 2005, Las Vegas USA.

Giannakidou, A. 2005. N-words and the Negative Concord. In M. Everaert, H. Van Riemsdijk, R. Goedemans and B. Hollebrandse (eds), The Blackwell Companion to Syntax, Vol III, Oxford: Blackwell.

Lehrndorfer A. 1996. Kontrolliertes Deutsch: Linguistische und Sprachpsychologische Leitlinien fuer eine (maschniell) kontrollierte Sprache in der technischen Dokumentation, Tuebingen : Narr.

Hatim, B. 1997. Communication Across Cultures: Translation Theory and Contrastive Text Linguistics, University of Exeter Press. 